\title{
Collisions of quasi-one-dimensional solitons in triangular Morse lattice
}

\author{
A. Chetverikov ${ }^{1, \dagger}$, W. Ebeling², M. G. Velarde ${ }^{3}$ \\ †chetverikovap@info.sgu.ru

\begin{abstract}
${ }^{1}$ Department of Physics, Saratov National Research State University, Astrakhanskaya 83, 410012 Saratov, Russia
${ }^{2}$ Institut fur Physik, Humboldt Universitat Berlin, Newtonstrasse 15, 12489 Berlin, Germany

${ }^{3}$ Instituto Pluridisciplinar, Universidad Complutense, Paseo Juan XXIII, 1, 28040 Madrid, Spain
\end{abstract}

We study by means of numerical simulation collisions of quasi-one-dimensional solitonic excitations in a $2 \mathrm{D}$ lattice of particles interacting via Morse potential forces. Local mobile excitations arise as a result of strong kicks for one or some selected particles stimulating motion of compression of density along crystallographic axes. It is shown that both two colliding head-on excitations and two excitations moving in parallel rods behave as real solitons and do not deform after contact with each other. Excitations moving in non-parallel axes collapse while meeting each other in the same point. But only one of them is destroyed if it crosses a trajectory of other local excitation just after passing of the latter because of temporal local deformation of a lattice behind a solitonic excitation. This effect provides possibility of control of motion of solitons and may be used for control of soliton assisted transport of charged particles.

Keywords: anharmonic lattice dynamics, Morse interaction, triangular lattice, simulations, quasi-one-dimensional solitons, collisions of parallel and non-parallel moving solitons

\section{Introduction}

In several previous papers [1 -9] we studied the behavior of local mobile supersonic soliton-like excitations in onedimensional lattice of interacting particles focusing on problems of soliton-assisted transport of charges. The particles dynamics was treated classically always. The lattice interactions were of Toda or Morse type akin to the Lennard-Jones interaction, hence allowing for phonon and soliton - vibrations with compressions governed by the repulsive part of the potential. Here we concentrate on $2 \mathrm{D}$-systems. Solitons on a $2 \mathrm{D}$ rectangular lattice were studied in [10] and in [11-12]. Soliton-like excitations in triangular lattice were found in simulations in [13-15, 11-12]. Both horseshoe-like and high-energetic quasione-dimensional solitons with a high life-time have been observed. Note also that the simulations earlier performed suggested the existence of (thermal) solitons in 2D lattices $[16,17]$. We proceed here with study of dynamics of triangular lattices and concentrate on collisions of high-energetic quasi-one-dimensional solitons in both cases - moving along the same rod or parallel rods of crystallographic axes and moving ones along rods oriented not parallel to each other.

\section{Molecular-dynamical simulations of two-dimensional lattices with solitonic excitations and visualization}

We study a Hamiltonian model for particles with interactions via finite-range Morse potential

$$
\begin{gathered}
H_{\text {lattice }}=\sum_{n, k}\left\{\frac{p_{n}^{2}}{2 M}+V_{M}\left(r_{n}-r_{k}\right)\right\} \\
V_{\mathrm{M}}=D \cdot(\exp [-b(r-\sigma)]-2) \cdot \exp [-b(r-\sigma)]
\end{gathered}
$$

Here $M$ denotes the mass of a lattice particle, $r_{\mathrm{n}}, p_{n}, r=$ $\left.r_{\mathrm{n}}-r_{\mathrm{k}}, n, k=1, \ldots, N\right)$ describe their respective coordinates and momenta, $b$ characterizes the stiffness of the springlike constant in the Morse potential, and $D$ is the depth of the potential well, $\sigma$ defines equilibrium lattice spacing (see [18]). The finite-range Morse potential was used because of some numerical advantages, due to the cutoff at $1.5 \sigma$. To have dimensionless variables we consider in the following the spatial coordinates rescaled with $\sigma$ as unit length. Time is normalized to the inverse frequency of linear oscillations near the minimum of the potential well, $\omega_{0}$, whereas energy and temperature are scaled with twice the depth of the well $2 D$. In our simulations we consider systems of a few hundred atoms on a plane. Coordinates and velocities of particles are obtained by solving the equations of motion of each particle under the forces of interaction. Our simulation algorithm corresponds to a molecular dynamics code, i.e. the particles are not fixed to any lattice node but may move freely through the system, exchanging places with neighbors etc. Rather than using Cartesian coordinates $x$ and $y$, we use complex coordinates $Z=x+i y$. Then the classical Newton deterministic equations corresponding to the lattice Hamiltonian (1)

$$
\ddot{Z}_{n}=\sum_{k} F_{n k}\left(\left|Z_{n k}\right|\right) z_{n k}
$$


where

$$
\begin{gathered}
Z_{n k}=Z_{n}-Z_{k}, \quad F_{n k}\left(\left|Z_{n k}\right|\right)=-\left.\frac{d U^{M}(r)}{d r}\right|_{r=\left|Z_{n k}\right|}, \\
Z_{n k}=\frac{Z_{n}-Z_{k}}{\left|Z_{n}-Z_{k}\right|}=\frac{Z_{n k}}{\left|Z_{n k}\right|},
\end{gathered}
$$

are solved.

In more recent work we developed a method of visualization of solitons in nonlinear 2D-lattices [12-15]. We modeled the atoms as little spheres with "cores" represented by a Gaussian distribution centered at each lattice site:

$$
\rho(Z, t)=\sum_{\left|Z-Z_{n}(t)\right|<1.5} e^{-\frac{\left(Z-Z_{n}(t)\right)^{2}}{2 \lambda^{2}}} .
$$

Using data about trajectories of particles $Z_{\mathrm{n}}(t)$ and their velocities we can calculate the lattice atom distribution $\rho$ $(Z, t)$ at the instant $t$. To make the picture much clear we introduce an "extra density" $\rho_{\mathrm{ex}}=\rho-\rho_{\mathrm{c}}$ with $\rho_{\mathrm{c}}=1-\delta$, $|\delta| \ll 1$. Furthermore, to clearly distinguish moving "local compressions" we use $\rho_{\mathrm{ac}}=\sum_{\mathrm{k}} \rho_{\mathrm{ex}}\left(k t_{\mathrm{ac}}\right)$, where $t_{\mathrm{ac}}$ is the time interval for producing $\rho_{\mathrm{ex}}(x, y, k t)$, with $k=1,2, \ldots$

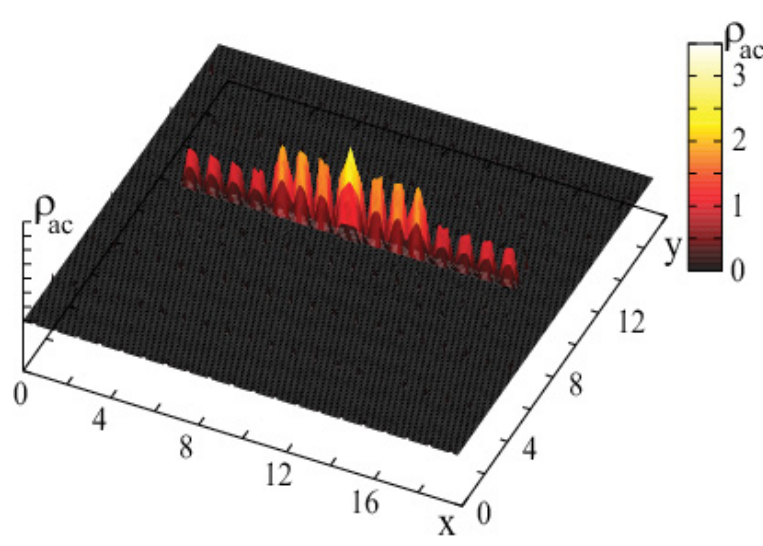

a)

\section{Collisions of quasi-one-dimensional solitons}

We consider here only dynamics of high-energetic quasione-dimensional solitons. Each of them is excited by a strong kick of one particle at initial time: $v_{\mathrm{m}}(0)=v_{\mathrm{m} 0}, q_{\mathrm{m}}$ $(0)=0$. Here only two solitons are supposed to be excited to take part in collisions, initial velocities $v_{\mathrm{m} 0}$ are supposed to be directed along one of crystallographic axes of a triangular lattice. Values of initial velocities must be strong enough to prevent a lateral phonon radiation because a spectrum of fast moving particles locates above the phonon band of the lattice [11], like in breather-like excitations [19]. Such excitations are localized in the one rod and behave as real solitons at head-on collisions. In Fig.1 one may observe two solitons, excited in a one rod with opposite directions of initial velocity, pass through each other without visible deformation and proceed to move as independent excitations in spite of some local deformation of a lattice behind each of them ("deformation tail").

Also solitons do not "feel" each other if they meet while running at adjacent parallel rods (Fig. 2). Tracks and density

Fig. 1. (Color online) Head-on collisions of solitons. Two identical high-energy solitons are excited by strong kicks (velocity $v_{0}=1.8$ in units $\omega_{0} \sigma$, energy $0.5(b \sigma)^{2} v_{0}^{2}$ in units $2 D$ ) imposed to two lattice particles in the same rod at the left and right borders, oppositely each other. Tracks of the excitations (in "bubble chamber representation", $\rho_{\mathrm{ac}}$ ) of the running solitons density are represented in a cumulative sequence of snapshots (a) as time proceeds during the time interval $\Delta t=4$ and density of the atomic cores (b) after passing solitons through each other is presented as a snapshot at time $t=4$ after launching the solitons. Parameter values: $N=400, b \sigma=4, \lambda=0.3$.

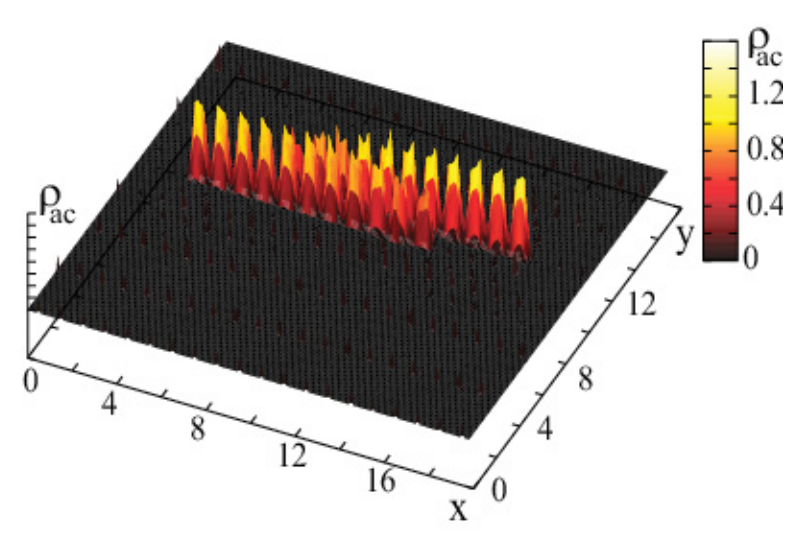

a)

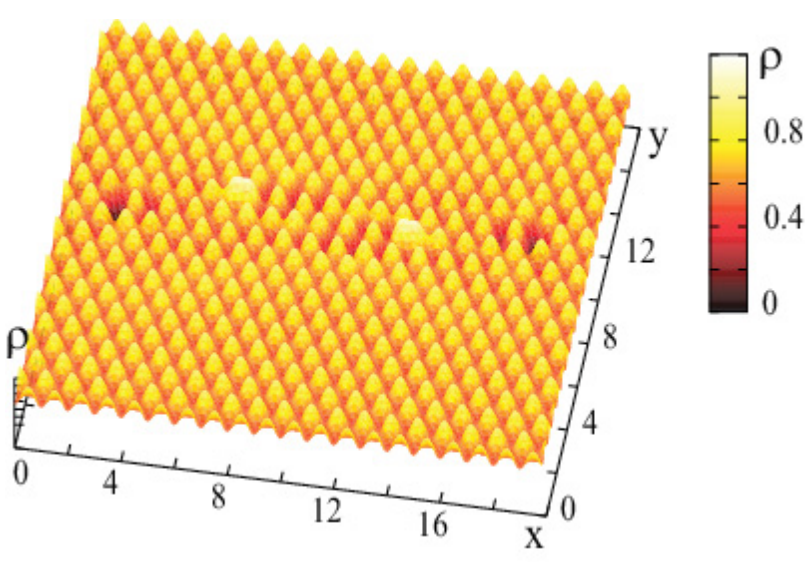

b)

Fig. 2. (Color online) Interaction of solitons running in parallel adjacent rods. Two identical high-energy solitons are excited by strong kicks imposed to two lattice particles in adjacent rods at the left and right borders, oppositely each other. Figures a) and b) as in Fig. 1. Parameter values as in Fig. 1 as well. 
distribution of each of them look like those in a case if each of them has been excited separately (here is not shown). It proves that perturbations induced by fast moving soliton in adjacent rods are really negligibly weak. Note that such behavior has been shown for quasi-one-dimensional breathers [19].

However the other results of collisions are observed if solitons run in non-parallel rods. We considered interaction of two identical, for simplicity, solitons. One had initial velocity $v_{10}=v_{1 \mathrm{x} 0}=v_{0}$ and moved in the beginning along an axis $x$. The other one was excited at $t=0$ in a rod lower than the first rod and has initial velocity $v_{20}=v_{0}\left(\cos 60^{\circ}+i \sin 60^{\circ}\right)$ moving in the beginning along one of two other axes of a lattice and then crossing a trajectory of the first soliton (Fig. 3,4). Results of interaction of solitons depend on their positions relatively each other at the instant when the second one (for certainty) crosses a trajectory of the first soliton. If they meet at the same point both of them crash (Fig.3). Energy of both excitations scatters in all directions, preliminary along crystallographic axes, invoking temporal local perturbation of a lattice like local melting. Spreading wave of a horse-shoe form scatters energy in increasing area and a lattice returns to the initial state after some relaxation (here is not shown).

However if the second soliton crosses the trajectory of the first one behind it the second soliton crushes but the first one proceeds to run as it moves alone (Fig.4 a,b). Oppositely, if the second soliton rushes just before the first one it keeps its

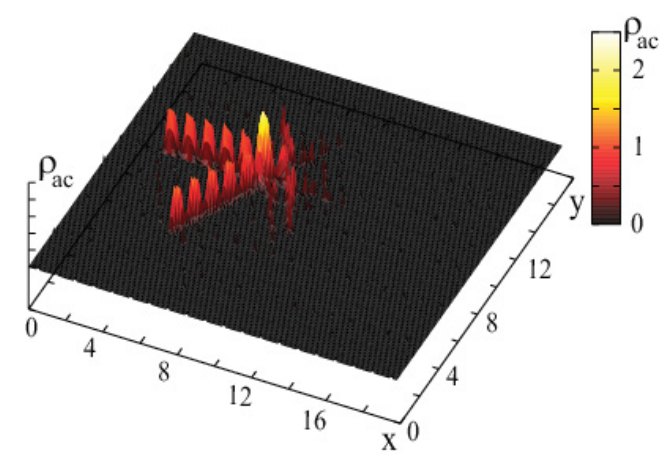

a)

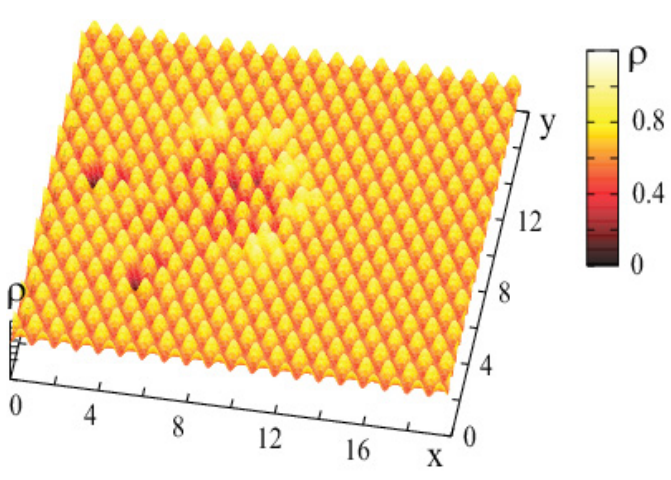

b)

Fig. 3. (Color online) Collapse of two solitons running in non-parallel rods at impact. Two identical high-energy solitons are excited by strong kicks imposed to two lattice particles in rods parallel to different crystallographic axes, one excited at the left border and other close to the lower border. Figures a) and b) and parameter values as in Fig. 1.

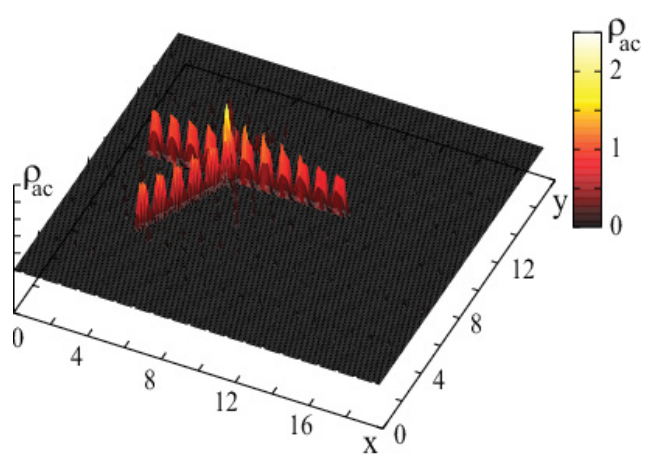

a)

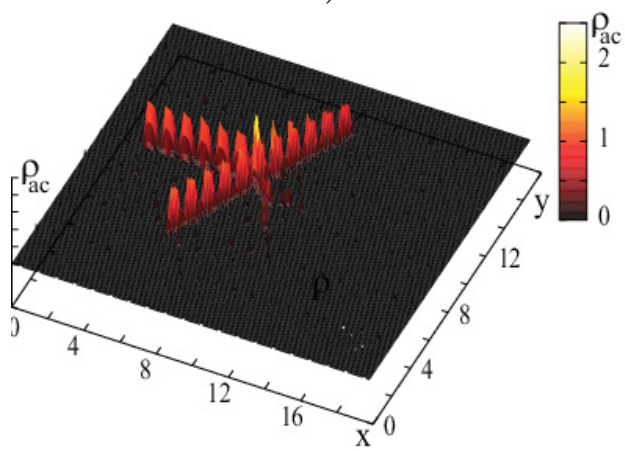

c)

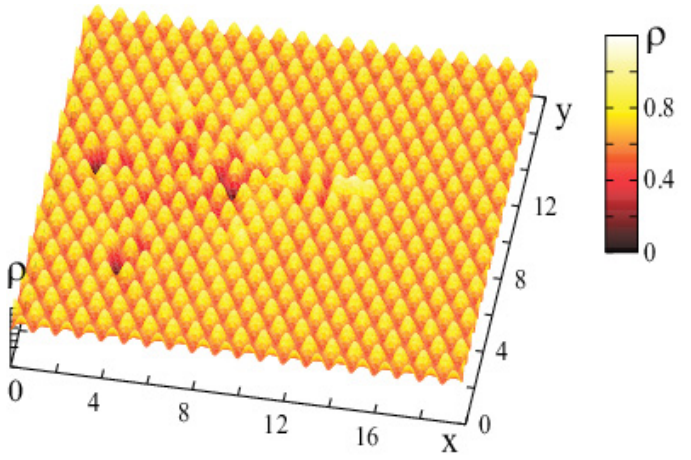

b)

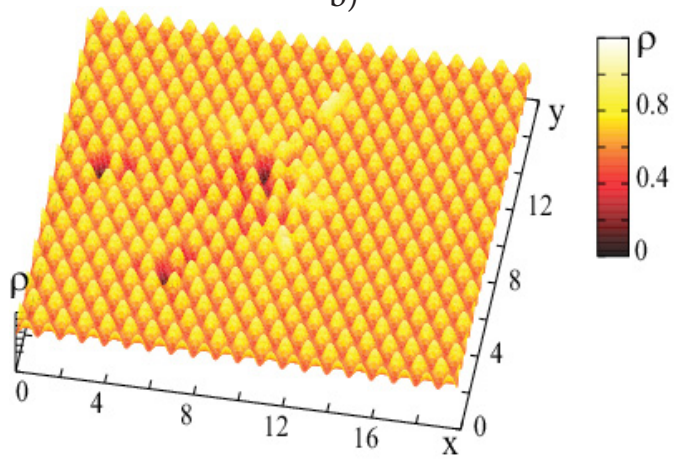

d)

Fig. 4. (Color online) Collapse of a soliton crossing a "tail" of a soliton running in non-parallel rod. Two identical high-energy solitons are excited by strong kicks imposed to two lattice particles in rods parallel to different crystallographic axes, one (the first) excited at the left border and other (the second) close to the lower border. In two upper panels the second soliton crosses a "tail" of the first one and collapses. In two lower panels the first soliton crosses a "tail" of the second one. Figures a) and c) are like Fig.1a, figures b) and d) are like Fig.1b. Parameter values as in Fig.1. 
motion but the first soliton crushes (Fig. 4c,d). In both cases a disturbance of the lattice in a "tail" of moving soliton is enough to break conditions to move for other soliton crossing a trajectory of the first of them. Similar behavior of solitons is observed if a second soliton moves along other axis with an angle $120^{\circ}$ to the $x$ axis (here is not shown). Such effect may be used for controlling solitonic transport when an electron carried by one of the solitons may be intercepted by another soliton to change direction of its motion and to deliver it to a chosen drain. Evidently, there are limitations for realization of the effects presented in Fig.4 (it may be supposed that behavior of solitons under interaction depends on initial energy of solitons, stiffness of bound between particles, a minimal distance between solitons under their interaction, temperature of a lattice) but we leave these questions for future study.

\section{Conclusion}

It is shown that quasi-one-dimensional soliton-like excitations in a triangular Morse-lattice behave as real solitons under collisions head-on and under running in parallel rods, they do not deform each other and proceed moving after contact between them. However under travelling in nonparallel rods they both collapse if colliding or only one of them destroys crossing a "tail" of the other one. The last suggests the possibility to control solectron motion (electron bound to a soliton).

Acknowledgements. The present work was supported by the Russian Foundation for Basic Research, grant RFBR14-52-12002.

\section{References}

1. M.G. Velarde, W. Ebeling, A.P. Chetverikov. Int. J. Bifurc.\&Chaos. 15, 245 (2005)

2. V.A. Makarov, M. G. Velarde, A. Chetverikov, W. Ebeling. Phys. Rev E. 73, 066626 (2006)
3. D. Hennig, A. Chetverikov, M. G. Velarde, W. Ebeling. Phys. Rev. E. 76, 046602 (2007)

4. M. G. Velarde, W. Ebeling, A.P. Chetverikov, D. Hennig. Int. J. Bifurc.\&Chaos. 18, 521 (2008)

5. D. Hennig, M. G. Velarde, W. Ebeling, A. Chetverikov. Phys. Rev. E. 78, 066606 (2008)

6. W. Ebeling, M. G. Velarde, A.P. Chetverikov. Condensed Matter Phys. 12, 633 (2009)

7. M. G. Velarde, A.P. Chetverikov, W. Ebeling, D. Hennig, J. J. Kozak. Int. J. Bifurc.\&Chaos. 20,185 (2010)

8. L. Brizhik, A.P. Chetverikov, W. Ebeling, G. Ropke \& M. G. Velarde. Phys. Rev. B. 85, 245105 (2012)

9. M. G. Velarde, A. P. Chetverikov, W. Ebeling, E. G. Wilson, K. J. Donovan. EPL. 106, 27004 (2014)

10. T. Yu. Astakhova, G. A. Vinogradov. J. Phys. A: Math. Gen. 39, 3593 (2006)

11. A.P. Chetverikov, W. Ebeling, M.G. Velarde. Eur. Phys. J. ST 222, 2531 (2013)

12. A. P. Chetverikov, W. Ebeling, M. G. Velarde. In.: Quodons in mica: nonlinear localized travelling excitations in crystals, ed. J.F. R. Archilla, N. Jiménez, V. J. SánchezMorcillo, L. M. García-Raffi, Springer Series in Materials Science. 221, 321. Springer, Berlin (2015)

13. A.P. Chetverikov, W. Ebeling, M.G. Velarde. Eur. Phys. J. B. 80, 137 (2011)

14. A.P. Chetverikov, W. Ebeling, M. G. Velarde. Physica D. 240, 1954 (2011)

15. A.P. Chetverikov, W. Ebeling, M.G. Velarde. Wave Motion. 48, 753 (2011)

16. W. Ebeling, A. A. Valuev, V. Podlipchuk J. Molecular Liquids. 73/74, 445 (1997)

17. W. Ebeling, U. Erdmann, J. Dunkel, M. Jenssen, J. Stat. Phys. 101, 443 (2000)

18. W. Ebeling, I. Sokolov. Statistical thermodynamics and stochastic theory of nonequilibrium systems. Singapore, World Scientific. (2005)

19. A.A. Kistanov, S.V. Dmitriev, A.P. Chetverikov, M. G. Velarde. Eur. Phys. J. B. 87, 211 (2014) 